\title{
Quasi-Static and Dynanmic Deformation Behaviors of Medium-Carbon Steels in a Wide Temperature Range
}

\author{
Byoungchul Hwang \\ Ferrous Alloys Group, Korea Institute of Materials Science, Changwon, Korea. \\ Email: entropy0@kims.re.kr \\ Received December $10^{\text {th }}, 2010$; revised March 21 ${ }^{\text {st }}$, 2011; accepted April $12^{\text {th }}, 2011$.
}

\begin{abstract}
This paper presents a study of the quasi-static and dynamic deformation behaviors of conventional and microalloyed medium-carbon steels in a wide temperature range. As strain rate increased, the flow stress increased at room temperature, but occasionally did not at elevated temperatures. The flow stress of the microalloyed steel containing precipitates was less sensitive to strain rate at room temperature than that of the conventional steel due to a relatively larger activation length. Microstructural observation of the steels deformed after compression test indicated that inhomogeneous deformation became more serious with increasing strain rate and temperature without fracturing in the highly localized region.
\end{abstract}

Keywords: Quasi-Static, Dynamic, Deformation Behavior, Medium-Carbon Steel, Precipitate

\section{Introduction}

Structural materials for automotive and machinery components have frequently obtained the required mechanical properties by heat-treating medium-carbon steels. In order to reduce energy consumption by eliminating heattreatment steps, therefore, steady attention on nonheat-treated steels has been paid for several decades. Since the 49MnVS3 medium-carbon steel microalloyed with $\mathrm{V}$ was first developed, non-heat-treated steels have been extensively applied to machinery and automotive components due to a good combination of strength and toughness [1]. Presently, both the non-heat-treated and heat-treated microalloyed medium-carbon steels where stable carbon-nitride precipitates mainly form by adding $\mathrm{V}, \mathrm{Ti}$, and $\mathrm{Nb}$ have been widely used as a substitute for conventional heat-treated medium-carbon steels.

Although many researchers have reported the mechanical properties of medium-carbon steels under quasi-static loading, the quasi-static and dynamic deformation behaviors have not been systematically compared over a wide temperature range, especially in conventional and microalloyed medium-carbon steels. Since the steels are highly likely to be subject to high-speed deformation during their manufacture and application, they are required to acquire information on dynamic deformation behavior [2-4]. For example, plastic deformation can become highly localized under the dynamic loading, which is often called an adiabatic shear band. In this localized area, the load-carrying capability seriously deteriorates and cracks nucleate, thereby causing eventual failures during high-speed processing.

In the present study, quasi-static and dynamic compressive tests were conducted on medium-carbon steels using a universal testing machine and a compressive Kolsky bar, and then the test results were analyzed in terms of microstructure, strain rate, and temperature. The aim of this study is to understand the effect of various factors on quasi-static and dynamic deformation behaviors of the medium-carbon steels and the associated mechanism.

\section{Experimental Procedures}

The materials used in this study were four kinds of medium-carbon steels fabricated with different chemical composition and heat-treatment conditions and were supplied by Caterpillar Inc., IL, USA. Two kinds are conventional SAE 1045 steels, and the others are V-microalloyed SAE 15V45 steels. Table 1 compares the composition range of the 15V45 steels with that of 
the 1045 steels. The $15 \mathrm{~V} 45$ steels are microalloyed with $\mathrm{V}$, and its Mn content is approximately doubled to that of the conventional 1045 steel. Both steels were properly heat-treated by quenching \& tempering (Q\&T) or austempering in order to achieve a good combination of strength and toughness. For convenience, they are referred to as '1045T', '1045S', '15V45T', and '15V45S' in this article, where the last letters ' $\mathrm{T}$ ' and ' $\mathrm{S}$ ' indicate $\mathrm{Q} \& \mathrm{~T}$ and austempering heat-treatments, respectively.

Compression tests were performed on them at low strain rates of $0.001,0.01$, and $0.1 \mathrm{sec}^{-1}$ using a universal testing machine. Tests performed at these strain rates are referred to as quasi-static in this article. The cylindrical specimens with $5.8 \mathrm{~mm}$ diameter and $6.3 \mathrm{~mm}$ length were machined from larger cylindrical disk using an electrostatic discharge machine so as to minimize the microstructure change. Specimen ends were lightly lubricated with a silicon grease before testing to reduce friction and ensure a flat surface.

A compressive Kolsky bar [2] was also used to test the specimens at high strain rates above $1000 \mathrm{sec}^{-1}$. Tests performed at these strain rates are referred to as dynamic in this article. The used specimens were cylindrical just as the ones used for quasi-static compression tests. The specimen situated between incident and transmission bar was compressed by a steel projectile projected at a high speed using argon gas pressure, and the strain rate could be controlled by varying the length of a steel projectile and the compressive gas pressure. During the dynamic compression, the incident, reflected, and transmitted pulses were respectively detected at strain gages, and recorded at an oscilloscope. Among the recorded signals, average compressive strain expressed as a function of time was measured from the reflected signal, while compressive stress expressed as a function of time was measured from the transmitted signal. Dynamic compressive stress-strain curves were obtained from these two parameters by eliminating the time term. Both quasi-static and dynamic compression tests were conducted over a temperature range of room temperature $\left(25^{\circ} \mathrm{C}\right)$ to $400^{\circ} \mathrm{C}$. Their microstructures were examined before and after deformation by an optical microscope.

\section{Results}

\subsection{Undeformed Microstructure}

Figures 1(a) and (b) are optical micrographs of the $15 \mathrm{~V} 45 \mathrm{~T}$ and 15V45S steels fabricated with different heat-treatment conditions for the microalloyed 15V45 steels. The quenched and tempered steel (15V45T steel) had typical fine-grained tempered martensite microstructure, whereas the austempered steel (15V45S steel) showed conventional upper bainite structures. In all
Table 1. Composition of the conventional (SAE 1045) and microalloyed0 (SAE 15V45) medium-crabon steels (wt\%).

\begin{tabular}{cccccc}
\hline & C & Mn & P & S & V \\
\hline SAE & $0.43 \sim 0.50$ & $0.60 \sim 0.90$ & $<0.04$ & $<0.05$ & - \\
1045 & & & & & \\
SAE & $0.43 \sim 0.50$ & $1.35 \sim 1.65$ & $<0.04$ & $<0.05$ & $0.10 \sim 0.25$ \\
$15 V 45$ & & & & & \\
\hline
\end{tabular}

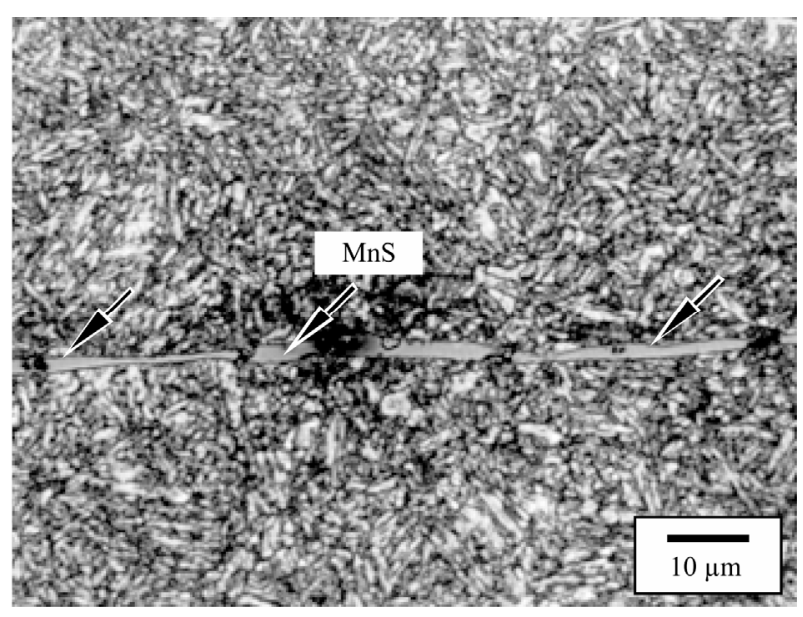

(a)

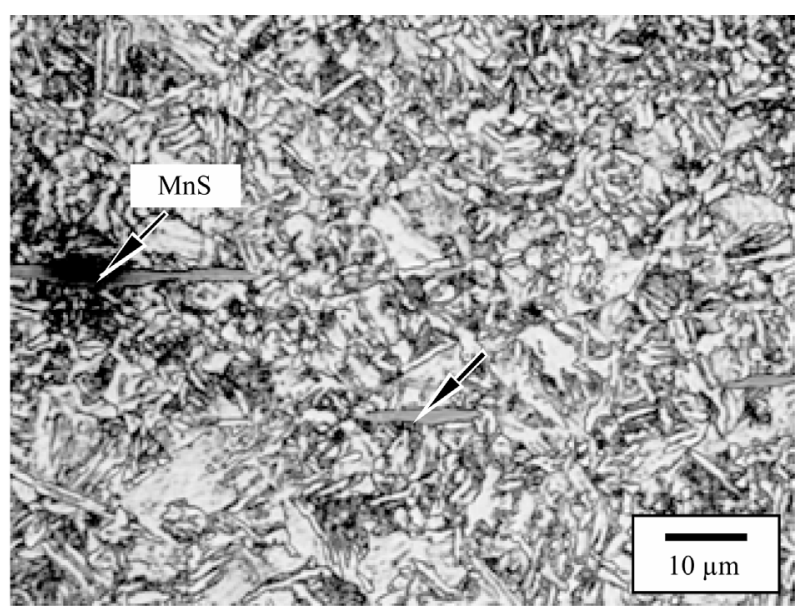

(b)

Figure 1. Optical micrograph of the (a) $1045 \mathrm{~T}$ and (b) 10V45S steels. Arrows indicate MnS inclusions. Nital etched.

steels, elongated MnS inclusions were present in the form of globular inclusions and stringers, and were mostly aligned along the rolling direction. The microalloyed 15V45 steels seemed to have finer microstructure than the conventional 1045 steels.

\subsection{Quasi-Static and Dynamic Stress-Strain Curves}

Figures 2(a) through (d) present the quasi-static and 


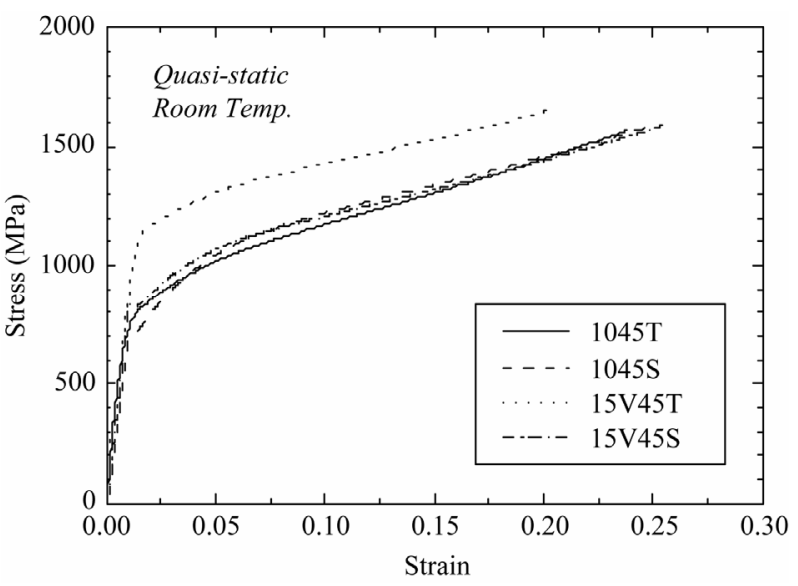

(a)

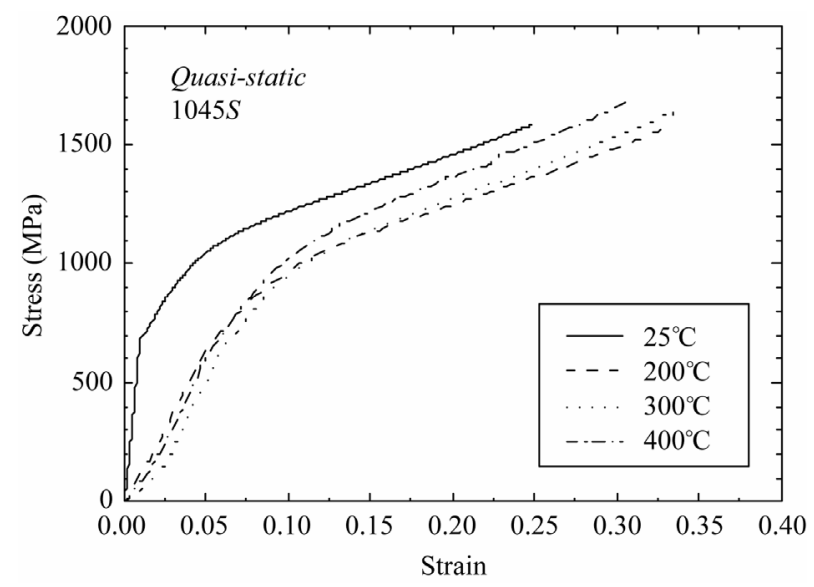

(c)

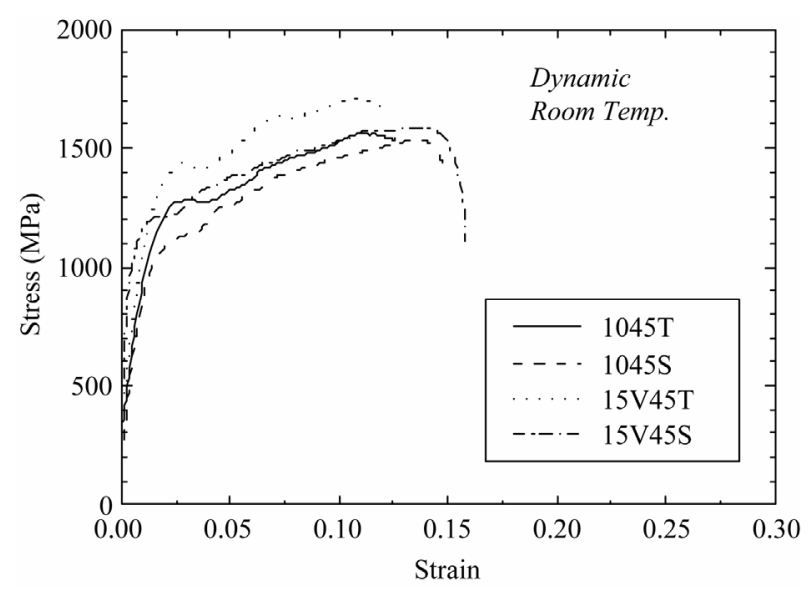

(b)

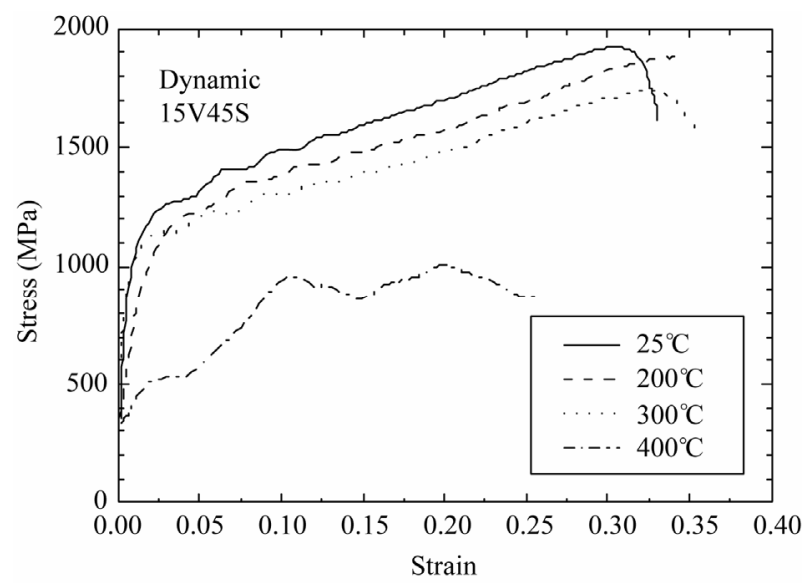

(d)

Figure 2. (a) and (b) Quasi-static and (c) and (d) dynamic compressive stress-strain curves of the steels tested at temperatures from room temperature to $400^{\circ} \mathrm{C}$.

dynamic compressive stress-strain curves of the steels tested at various temperatures. In dynamic tests, the total plastic strain was relatively smaller than that obtained in the quasi-static tests due to the constraint in the design of Kolsky bar. The stress under quasi-static compression was continuously increased with strain, whereas maximum stress appeared under dynamic compression because of the softening of material by local heating occurring during dynamic loading [2].

In the steels tested at room temperature, the microalloyed 15V45 steels were relatively higher flow stress than the conventional 1045 steels under both quasi-static and dynamic loadings, which resulted from the increased amount of manganese and the precipitation of vanadium carbides. Figures 2(c) and (d) are the quasi-static and dynamic compressive stress-strain curves of the 1045S and 15V45S steels tested at temperatures from room temperature to $400^{\circ} \mathrm{C}$. The plastic deformation and flow stress of materials are generally affected by test tem- perature [2,5]. Since the flow stress expectedly decreased with increasing temperature, the experimental results of the $1045 \mathrm{~S}$ and $15 \mathrm{~V} 45 \mathrm{~S}$ steels showed typical behavior of body-centered cubic (bcc) materials.

\subsection{Deformed Microstructure}

Figure 3(a) shows overall micrographs of the $1045 \mathrm{~S}$ steel dynamically deformed at an elevated temperature. Examination of the cross-section of the deformed steels showed a non-uniform deformation, which was more visible in the middle region between corners and center of the cross-section, whereas adiabatic shear bands were not observed. The degree of heterogeneity became serious as strain rate or temperature increased. $\mathrm{MnS}$ inclusions were mostly aligned along the deformation flow direction and some of them were severely distorted in heavily deformed region (Figure 3(b)).

In the specimens deformed at lower strain rates or room temperature, however, MnS inclusions remained 


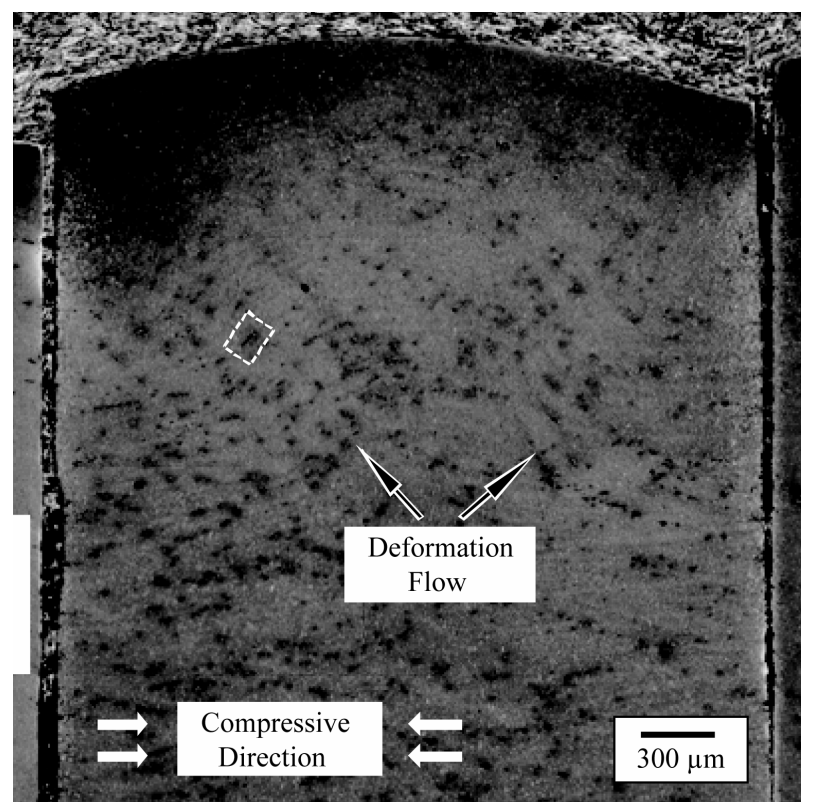

(a)

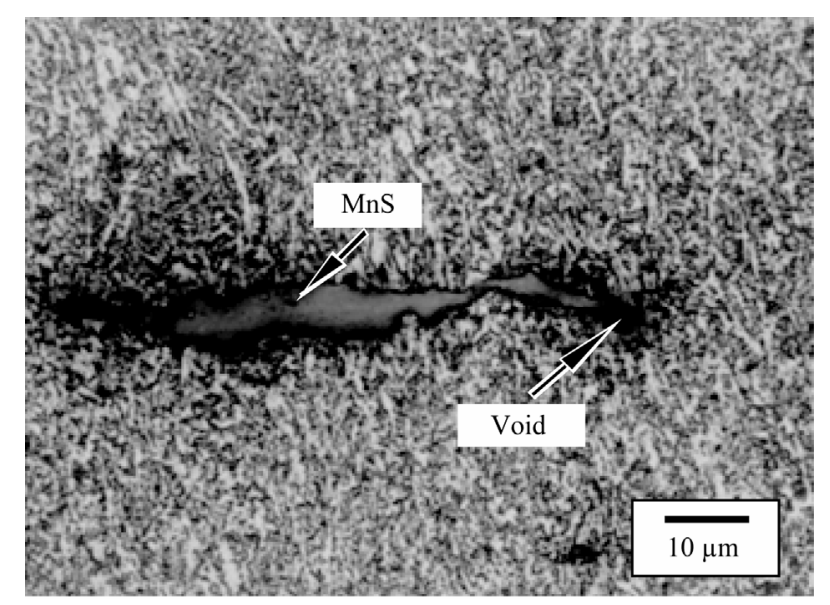

(b)

Figure 3. Optical micrograph of the $1045 \mathrm{~S}$ steel deformed dynamically at an elevated temperature at a high strain rate and (b) is a magnification of (a), showing a heavily distorted MnS inclusion and the formation of voids.

slightly elongated and aligned along the rolling direction as with those in the undeformed steels, and their shapes did not change much. The distortion of MnS inclusions was largely affected by strain rate than by test temperature.

\section{Discussion}

In general, it has been well recognized that the flow stress slightly increases with logarithmic strain rate at low strain rates, while it more rapidly increases with logarithmic strain rate at high strain rates [2,4-6]. Figure 4(a) provides the effect of strain rate on the $5 \%$ flow stress of the steels tested at room temperature. The flow stress usually increased with the strain rate, and thus dynamic flow stress was higher than quasi-static flow stress. Although the difference in flow stresses reflected scatter for some specimens, the flow stress showed more increased strain-rate dependency at high strain rates than at low strain rates. Campbell and Ferguson [6] reported from a study of the mechanical properties of mild-carbon steel tested at strain rates from $10^{-3}$ to $4 \times 10^{4} \mathrm{sec}^{-1}$ that the shear flow stress was in linear proportion to the value of strain rate on logarithmic scale at strain rates below $10^{2} \mathrm{sec}^{-1}$, and found that the strain-rate sensitivity of the flow stress was a decreasing function of temperature. Lee and Liu [4] obtained the dynamic flow stress of mildand medium-carbon steels at high strain rates between $10^{3}$ and $10^{4} \mathrm{sec}^{-1}$, and confirmed that the magnitude of the flow stress increased significantly with increasing logarithmic strain rate at the high strain rates. In the steels investigated, also, the strain-rate dependency of the flow stress was relatively prominent at high strain rates than at low strain rates, which indicates that the present data results are in agreement with previous investigations [2-6]. It should be noted that this behavior at low strain rates is ascribed to the thermal activation in which dislocations require thermal energy to overcome obstacles such as precipitates by cross slip, whereas there are still many controversies over strain-rate controlling mechanisms at high strain rates [2,5,7].

Figures 4(b) and (c) present the effect of strain rate on the flow stress of the $1045 \mathrm{~S}$ and $15 \mathrm{~V} 45 \mathrm{~S}$ steels tested at various temperatures of room temperature to $400^{\circ} \mathrm{C}$. The flow stress of the $1045 \mathrm{~S}$ steel was more largely affected by strain rate at room temperature than that of the 15V45S steel having precipitates. The flow stress $\tau$ consists of two parts, a thermally activated part, $\tau_{t}$, and an athermal part, $\tau_{a}$. Only $\tau_{t}$ is influenced by variation in strain rate or temperature.

$$
\tau=\tau_{t}+\tau_{a}
$$

The following equation is often used to describe a relationship between strain rate, $\gamma$, and flow stress [8]:

$$
\begin{aligned}
\dot{\gamma} & =\dot{\gamma}_{0} \exp \left[-\frac{Q-V\left(\tau-\tau_{a}\right)}{k_{b} T}\right] \\
& =\dot{\gamma}_{0} \exp \left[-\frac{Q}{k_{b} T}+\frac{V \tau}{k_{b} T}-\frac{V \tau_{a}}{k_{b} T}\right] .
\end{aligned}
$$

Here, $\dot{\gamma}_{0}$ is a constant, $Q$ is the activation energy, $V$ is the activation volume, $k_{b}$ is Boltzmann's constant $(1.38 \times$ $10^{-23} \mathrm{~J} \cdot \mathrm{K}^{-1}$ ), and $T$ is an absolute temperature.

The activation volumes determined from the slopes in Figures 3(b) and (c) were $6.45 \times 10^{-28} \mathrm{~m}^{3}$ for the $1045 \mathrm{~S}$ steel and $7.82 \times 10^{-28} \mathrm{~m}^{3}$ for $15 \mathrm{~V} 45 \mathrm{~S}$ steel. The activation 


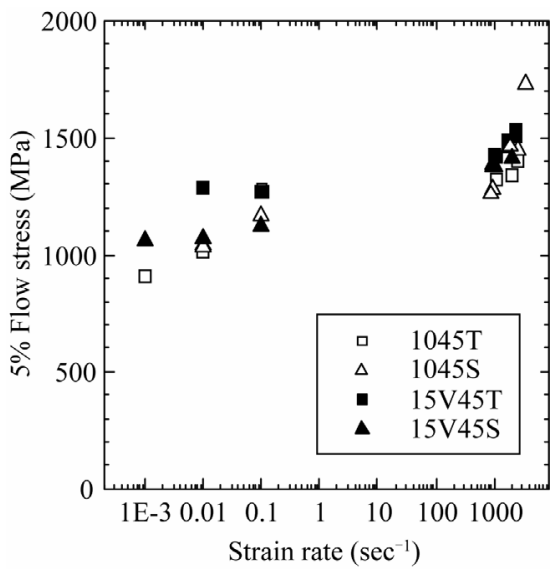

(a)

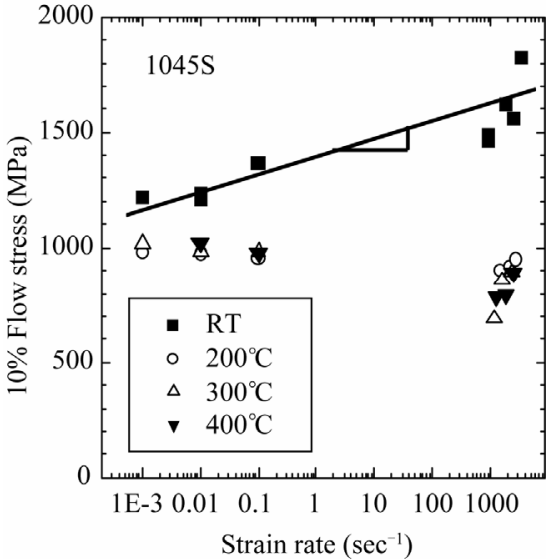

(b)

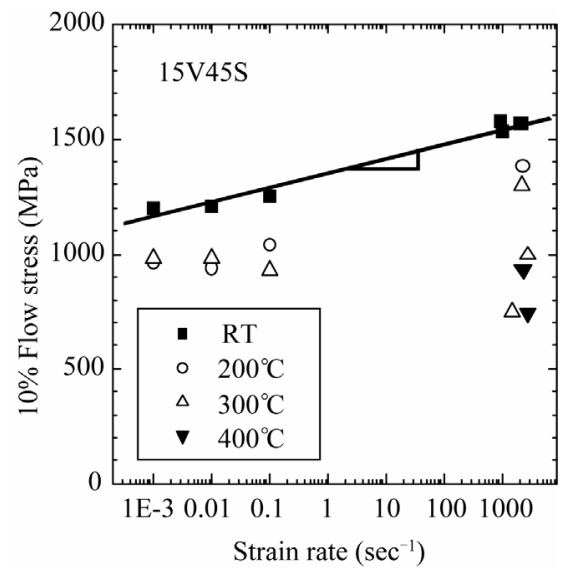

(c)

Figure 4. (a) $5 \%$ flow stress plotted as a function of strain rate for the steels tested at room temperature. (b) and (c) are $10 \%$ flow stress plotted as function of strain rate for the $1045 \mathrm{~S}$ and $15 \mathrm{~V} 45 \mathrm{~S}$ steels tested at temperatures up to $400^{\circ} \mathrm{C}$.

volume for pure iron is $3.8 \times 10^{-28} \mathrm{~m}^{3}$ [9]. Also, the activation volume is:

$$
V=b d l
$$

where $b$ is the Burgers vector, $d$ is the activation distance and $l$ is the activation length. In this case, $d$ is equal to $b$ the distance from on Peierls valley to the next.

As the Burgers vector is equal to $2.48 \times 10^{-10} \mathrm{~m}$ for bcc iron, the activation length of the $1045 \mathrm{~S}$ and $15 \mathrm{~V} 45 \mathrm{~S}$ steels is about $1.05 \times 10^{-8} \mathrm{~m}$ and $1.27 \times 10^{-8} \mathrm{~m}$, respectively. The 15V45S steel having precipitates has a larger activation length than the 1045S steel and thus lowers locally the Peierls stress. This result is consistent with the theory that precipitates interact with screw dislocations in bcc iron locally [5,8]. As a result of the lowered Peierls stress, the flow stress of the microalloyed 15V45S steel having precipitates is less sensitive to strain rate than that of the conventional 1045S steel that does not have precipitates (Figures 4(b) and (c)).

In the $1045 \mathrm{~S}$ and $15 \mathrm{~V} 45 \mathrm{~S}$ steels tested at temperatures over $200^{\circ} \mathrm{C}$, the flow stress did not clearly increase with increasing strain rate. As the strain rate increased, the flow stress remained constant or rather decreased unlike the results tested at room temperature. According to the Equation (2), the flow stress had a relatively lower strain-rate dependency at an elevated temperature than at room temperature. It is possibly attributed to the fact that a localized inhomogeneous deformation occurs by the increased temperature.

On the other hand, deformation after compression tests may be inhomogeneous and be concentrated into two bands that formed an X-shaped pattern on a polished section. In three dimensions, this pattern would be the surfaces of two cones of deformation with their apices in the center of the specimen $[7,10]$. Such deformation is typical of material that has undergone barreling during compression testing. At high-speed deformation of materials, adiabatic heating also may occur, where the heat generated during the deformation in a particular region is retained and causes a local rise in temperature [3,7]. Further deformation is concentrated preferentially in this zone and the process may continue until fracture occurs. One of the good examples of this phenomenon is the formation of adiabatic shear bands in HY-100 and AISI 4340 steels having a tempered martensite structure $[3,10]$, which is the same microstructure to the $1045 \mathrm{~T}$ and 15V45T steels. Observation results of the steels deformed under the current strain rates and temperatures, however, indicated that all the steels were not fractured after compression tests. Also, voids or cracks was hardly observed, but only a few isolated voids were occasionally formed without being connected to form a crack even in the steels dynamically deformed at elevated temperatures (Figure 3(b)). Consequently, the medium-carbon steels investigated in this study had the ability enough to withstand high-speed deformation at strain rates of up to 3000 $\mathrm{sec}^{-1}$ and temperatures from room temperature to $400^{\circ} \mathrm{C}$ without fracturing. This means that the medium-carbon steels are suitable materials for high-speed deformation processing.

\section{Conclusions}

The quasi-static and dynamic deformation behaviors of conventional and microalloyed medium-carbon steels were analyzed in terms of microstructure, strain rate, and temperature. The following conclusions can be drawn. 1) The flow stress increased with strain rate and was more strain-rate sensitive at high strain rates than at low strain rates, whereas it decreased with increasing temperature; 2) The flow stress of the 15V45S steel containing pre- 
cipitates was relatively less sensitive to strain rate at room temperature than that of the conventional 1045S steel that did not have precipitates because of the increased in activation length; 3) The quasi-static and dynamic deformation under the current strain rate and temperature were non-uniform that was typically found in compression tests, and the degree of heterogeneity increased as strain rate or temperature increased. Therefore, the present study provides a valuable reference for the application of these medium-carbon steels.

\section{Acknowledgements}

This work was sponsored by the Korea Research Foundation under Grant No. 2005-214-D00201. The author thanks Professor C. L. Briant of Brown University and C. Bissahoyo of Caterpillar Inc. for their help with the compressive dynamic Kolsky testing.

\section{REFERENCES}

[1] T. Gladman, “The Physical Metallurgy of Microalloyed Steels," 1st Edition, the Institute of Materials, London, 1997.

[2] M. A. Meyers, "Dynamic Behavior of Materials," John Wiley \& Sons, New York, 1994. doi:10.1002/9780470172278

[3] K. M. Cho, S. Lee, S. R. Nutt and J. Duffy, “Adiabatic Shear Band Formation during Dynamic Torsional Deformation of an HY-100 Steel,” Acta Materialia, Vol. 41, No. 3, 1993, pp. 923-932.
doi:10.1016/0956-7151(93)90026-O

[4] W.-S. Lee and C.-Y. Liu, "Comparison of Dynamic Compressive Flow Behavior of Mild and Medium Steels over Wide Temperature Range,” Metallurgical and Materials Transactions A, Vol. 36A, No. 11, 2005, pp. 31753186. doi:10.1007/s11661-005-0088-1

[5] M. A. Meyers, R. W. Armstron and H. O. K. Korchner, "Mechanics and Materials-Fundamentals and Linkages,” John Wiley \& Sons, New York, 1999.

[6] J. D. Campbell and W. G. Ferguson, "The Temperature and Strain Rate Dependence of the Shear Strength of Mild Steel,” Philosophical Magazine, Vol. 21, No. 169, 1970, pp. 63-82. doi:10.1080/14786437008238397

[7] Y. Bai and B. Dodd, "Adiabatic Shear LocalizationOccurrence, Theories and Applications,” Pergamon Press, New York, 1994.

[8] S. Vaynman, M. E. Fine, S. Lee and H. D. Espinosa, "Effect of Strain Rate and Temperature on Mechanical Properties and Fracture Mode of High Strength Precipitation Hardened Ferritic Steels,” Scripta Materialia, Vol. 55, No. 4, 2006, pp. 351-354. doi:10.1016/j.scriptamat.2006.04.029

[9] A. Smith, H. Luo, D. N. Hanlon, J. Sietsma and S. van der Zwaag, "Recovery Processes in the Ferrite Phase in C-Mn Steel,” ISIJ International, Vol. 44, No. 7, 2004, pp. 1188- 1194. doi:10.2355/isijinternational.44.1188

[10] A. G. Odeshi, M. N. Bassim, S. Al-Ameeri and Q. Li, "Dynamic Shear Band Propagation and Failure in AISI 4340 Steel,” Journal of Materials Processing Technology, Vol. 169, No. 2, 2005, pp. 150-155. doi:10.1016/j.jmatprotec.2005.03.016. 